\title{
openheart Work-life balance: a comparison of women in cardiology and other specialties
}

\author{
Anastasia Vlachadis Castles (1) , , ${ }^{1,2}$ Sonya Burgess, ${ }^{3,4,5}$ Kristy Robledo, ${ }^{6}$ \\ Anna L Beale (1) , ,,8,9 Sinjini Biswas, ${ }^{7,9}$ Louise Segan (1) ,7,10 Sarah Gutman (D) ,7,8,9 \\ Swati Mukherjee, ${ }^{11}$ Angeline Leet, ${ }^{7}$ Sarah Zaman (i) ${ }^{12,13}$
}

\begin{abstract}
- Additional supplemental material is published online only. To view, please visit the journal online (http://dx.doi.org/10.
\end{abstract} 1136/openhrt-2021-001678)

To cite: Vlachadis Castles A, Burgess S, Robledo K, et al. Work-life balance: a comparison of women in cardiology and other specialties. Open Heart 2021;8:e01678. doi:10.1136/ openhrt-2021-001678

Received 6 April 2021 Accepted 7 June 202

\section{Check for updates}

(c) Author(s) (or their employer(s)) 2021. Re-use permitted under CC BY-NC. No commercial re-use. See rights and permissions. Published by BMJ.

For numbered affiliations see end of article.

Correspondence to Associate Professor Sarah Zaman; sarah.zaman@sydney. edu.au

\section{ABSTRACT}

Objective Significant gender disparities exist in some medical specialties, particularly cardiology. We assessed work, personal life and work-life balance in women in cardiology in Australia and New Zealand (NZ), compared with other specialties, to determine factors that may contribute to the lack of women in the specialty. Methods This study is a prospective survey-based cohort study comparing cardiology and non-cardiology specialties. An online survey was completed by female doctors in Australia and NZ, recruited via email lists and relevant social media groups. The survey included demographics, specialty, stage of training, work hours/ setting, children and relationships, career satisfaction, income and perceptions of specialty.

Results 452 participants completed the survey (median age 36 years), of which 57 (13\%) worked in cardiology. Of all respondents, $84 \%$ were partnered and $75 \%$ had children, with no difference between cardiology and noncardiology specialties. Compared with non-cardiology specialties, women in cardiology worked more hours per week (median 50 hours vs 40 hours, $p<0.001$ ), were more likely to be on call more than once per week ( $33 \%$ vs $12 \%$, $\mathrm{p}<0.001$ ) and were more likely to earn an annual income $>\$ 300000$ (35\% vs $10 \%, p<0.001)$. Women in cardiology were less likely to agree that they led a balanced life (33\% vs $51 \%, p=0.03$ ) or that their specialty was female friendly $(19 \%$ vs $75 \%, p<0.001)$ or family friendly $(20 \%$ vs $63 \%$, $\mathrm{p}<0.001$ ).

Conclusions Compared with other specialties, women in cardiology reported poorer work-life balance, greater hours worked and on-call commitments and were less likely to perceive their specialty as female friendly or family friendly. Addressing work-life balance may attract and retain more women in cardiology.

\section{INTRODUCTION}

Significant disparities exist in the proportion of female and male doctors, at both the trainee and consultant level, in certain medical specialties, particularly in cardiology. ${ }^{1-4}$ Less than $15 \%$ of practicing cardiologists in Australia are female, and approximately $21 \%-22 \%$ of cardiology trainees are female $;^{25}$ this is mirrored across the developed world. ${ }^{346-8}$ Projections based on current trends predict that the low proportion of

\section{Key questions}

What is already known about this subject?

- Studies have demonstrated that the proportion of female doctors in the specialty of cardiology is much lower than in other physician specialties and most surgical specialties. However, there is a lack of data around the issues of work-life balance, flexibility and conduciveness to raising a family, in relation to cardiology compared with other specialties.

What does this study add?

- This study identifies major differences in the worklife balance of female doctors in cardiology compared with other specialties. Few studies assessing work-life balance have been undertaken in international cohorts with none performed in the Australian and New Zealand medical profession. This study demonstrates that women in cardiology report poorer work-life balance, greater hours worked and on-call commitments and a perception that their specialty is less female friendly or family friendly.

How might this impact on clinical practice?

- By identifying potential barriers to women pursuing a career in cardiology, we can develop strategies to address them, assisting in recruitment and retention of women within the male-dominated cardiology specialty.

female cardiologists is unlikely to change within the next few decades, ${ }^{2}$ despite equal gender proportions of medical graduates. ${ }^{9}$ In contrast, other specialties that are perceived as 'female friendly', such as paediatrics and obstetrics/gynaecology, have a predominance of female trainees. ${ }^{1}$ Diversity in culture, ethnicity and gender, specifically in medicine, has been recognised as providing an advantage in the workplace. ${ }^{10}{ }^{11}$ In cardiology in particular, improved guidelinedirected patient care has been seen with treatment by female physicians. ${ }^{12}$ Improving the gender gap at the professional level has been described as a powerful tool to improve cardiovascular outcomes in women. ${ }^{13}$

Work-life balance is a concept that is poorly and variably defined, and perhaps the 
difficulty in defining this concept reflects its complexity. A review of the construct of work-life balance by Kalliath and Brough gave several definitions, centering around the concepts of multiple roles, relationships between them and an individual's satisfaction in their roles. ${ }^{14}$ While work-life balance is an issue that is relevant for female and male physicians, it is likely to affect women more, as they are generally responsible for a disproportionate majority of household and childrearing tasks, compared with men. ${ }^{15}$

There are currently limited data available to determine the current perceptions of specialties in regard to worklife balance, flexibility and conduciveness to raising a family. Knowledge of these factors may assist in helping doctors choose a specialty pathway and identify barriers to women entering cardiology. This study aimed to explore work-life balance and work flexibility for female doctors in Australia and New Zealand (NZ), including family life, parenting and job satisfaction. In particular, we compared differences work-life balance between cardiology and non-cardiology specialties. By identifying work-life balance issues within cardiology, we ultimately aim to further our understanding of which strategies could be developed to improve diversity within this maledominated specialty.

\section{METHODS \\ Participants}

An anonymous, online survey was distributed via email and links posted in selected Facebook groups in September 2019. The survey was targeted at Australian and NZ female doctors across all specialties. The survey link was emailed to the Australian/NZ women in cardiology group (88 female cardiologists, trainees and fellows). A survey link was posted on the following medical Facebook groups: Royal Australasian College of Physicians (RACP) Trainees ( 4000 female physician trainees); Medical Mums/Mums to Be Australia/NZ ( 9000 medical mums) and the Women's Edition Doc-to-Doc group ( 9000 members). All Facebook groups were private/closed groups requiring evidence to be uploaded demonstrating medical registration (eg, Australian Health Practitioner Regulation certificate) or college identification (eg, RACP, Royal Australasian College of Surgeons). Respondents who self-identified as female and who completed the entire survey were included in the study.

Specialty was categorised as one of the following: cardiology, non-cardiology internal medicine (basic physician trainees, gastroenterology, general medicine, geriatrics, endocrinology, haematology, immunology, infectious diseases, nephrology, neurology, medical obstetrics, oncology, respiratory, rheumatology, sexual health), critical care (anaesthetics, emergency, intensive care), obstetrics/gynaecology, paediatrics, surgery, general practice and other (including addiction medicine, medical administration, palliative care, pathology, public health, radiology, rehabilitation medicine). Respondents were further categorised into trainees (undertaking specialty/ subspecialty training) or consultants (completed all training).

Data were stored in a centralised, secure online database housed within Monash University.

\section{Survey}

Participants completed a 62-item online survey, with questions related to: demographics (age, gender, ethnicity); career/training (specialty, stage of training, work hours and setting, income); personal life (relationship status, children, childcare); work/life balance and career satisfaction; mentoring and medical culture; perception of cardiology as a career and occupational radiation exposure. All questions were closed questions, with answers predominantly presented as choice of category/range, absolute agreement (yes/no/unsure) or level of agreement (modified 5-point Likert Scale).

\section{Patient and public partnership}

This research focused on the female physicians who provide care to patients and the public and did not involve patient or public participation. In this case, patient and public involvement was neither considered feasible nor appropriate for this study.

\section{Statistical analysis}

Data are summarised by area of specialty, and comparisons are made between cardiology versus other specialities, with prevocational doctors excluded from the cardiology/non-cardiology comparison. Associations in categorical variables were analysed with $\chi^{2}$ tests and expressed as number and percentage. Continuous variables were analysed with two-sample t-tests or Wilcoxon rank-sum tests and expressed as mean (SD) or median $(\mathrm{IQR})$ as appropriate. Mean agreement was calculated by assuming a linear scale of agreement $(1=$ strongly disagree to 5 =strongly agree). Comparisons between trainees and specialists for mean agreement were performed by fitting an interaction term in a linear model. Statistical analyses were performed using SAS V.9.4 and R V.4.0.2. ${ }^{16}$ $P$ values $<0.05$ were considered statistically significant and no adjustments have been made for multiple comparisons.

\section{RESULTS}

A total of 452 female doctors completed the survey. Respondents completed sections of the survey that were general or relevant to their circumstances (eg, relating to children). Respondent characteristics are shown in table 1. Median age was 36 years (IQR 32-41), and respondents were predominantly Caucasian $(366 / 450$, $81 \%)$ or Asian $(60 / 450,13 \%)$. Respondents were from a wide range of specialties, with the highest representation from non-cardiology adult internal medicine specialties $(117 / 452,26 \%)$ and general practice $(80 / 452,18 \%)$ and women in cardiology comprising $57 / 452(13 \%)$. A total of $55 \%$ of those who participated in the survey $(246 / 452)$ 
Table 1 Respondent characteristics according to specialty

\begin{tabular}{|c|c|c|c|c|c|c|c|c|c|c|}
\hline $\begin{array}{l}\text { Characteristic } \\
\text { Number (\%) unless } \\
\text { stated otherwise }\end{array}$ & Cardiology & $\begin{array}{l}\text { Critical } \\
\text { care }\end{array}$ & GP & O\&G & Paediatrics & $\begin{array}{l}\text { Internal } \\
\text { medicine- } \\
\text { other }\end{array}$ & Prevocational & Surgery & Other & Total \\
\hline Number of Respondents & 57 & 68 & 80 & 15 & 36 & 117 & 29 & 11 & 39 & 452 \\
\hline Age Median, (IQR) & $44(37,54)$ & $36(33,40)$ & $37(33,41)$ & $34(31,38)$ & $34(31,38)$ & $36(32,40)$ & $28(26,31)$ & $34(32,34)$ & $37(33,43)$ & $36(32,41$ \\
\hline \multicolumn{11}{|l|}{ Ethnicity } \\
\hline Caucasian & $50(88)$ & $57(84)$ & $67(84)$ & $12(80)$ & $28(78)$ & $89(76)$ & $22(76)$ & $33(85)$ & $8(73)$ & $366(81)$ \\
\hline Asian & $6(11)$ & $8(12)$ & $10(13)$ & $3(20)$ & 2 (6) & $20(17)$ & $4(14)$ & $6(15)$ & 1 (9) & $60(13)$ \\
\hline Indigenous & $0(0)$ & $0(0)$ & $1(1)$ & $0(0)$ & $1(3)$ & $0(0)$ & $0(0)$ & $0(0)$ & $0(0)$ & $2(0)$ \\
\hline Middle Eastern & $0(0)$ & $0(0)$ & $0(0)$ & $0(0)$ & $0(0)$ & $1(1)$ & $1(3)$ & $0(0)$ & $0(0)$ & $2(0)$ \\
\hline Other & $1(2)$ & $3(4)$ & $2(3)$ & $0(0)$ & $4(11)$ & $6(5)$ & $2(7)$ & $0(0)$ & $2(18)$ & $20(4)$ \\
\hline \multicolumn{11}{|l|}{ Stage of training } \\
\hline Training & $12(21)$ & $30(44)$ & $16(20)$ & $10(67)$ & $21(60)$ & $56(48)$ & $29(100)$ & $20(53)$ & $8(73)$ & $202(45)$ \\
\hline Specialist & $45(79)$ & $38(56)$ & $63(80)$ & $5(33)$ & $14(40)$ & $60(52)$ & $\mathrm{N} / \mathrm{A}$ & $18(47)$ & $3(27)$ & $246(55)$ \\
\hline
\end{tabular}

Due to the small numbers in each individual specialty group, statistical comparisons were performed for cardiology versus non-cardiology specialties in table 3 . GP, general practice; O\&G, obstetrics and gynaecology.

were consultants, having completed specialty training. The perception of cardiology as being significantly less 'female friendly' and 'family friendly' was similar between trainees and specialists (online supplemental figure 1).

\section{Personal lives of female doctors}

The comparison of women in cardiology with women in other specialties in regard to relationships and children is shown in tables 2 and 3 (table 3 excludes prevocational doctors). The majority of respondents were in a relationship $(84 \%, 356 / 423)$, with $27 \%(115 / 423)$ partnered with another doctor. Over two-thirds of respondents $(71 \%, 322 / 452)$ had children. Of those with children, almost all $(93 \%, 299 / 322)$ had their first child after completing their medical degree and most had their first child prior to completing specialty training or during a research phase of their career $(71 \%, 230 / 322)$. In regard to paid childcare, $53 \%(172 / 322)$ used day care and $23 \%$ (73/322) used a nanny. The majority had returned to work in a part-time clinical capacity after delivering a child $(54 \%, 174 / 322)$, while over one-third returned in a full-time clinical capacity $(34 \%, 109 / 322)$.

There was no significant difference in marital status (partnered, divorced/separated) between women in cardiology compared with other specialties (table 3 ). However, women in cardiology were more likely to be partnered with another doctor $(40 \%$ vs $27 \%$, $\mathrm{p}=0.002)$. There was no significant difference in proportion of women who did not have children in cardiology compared with non-cardiology specialties $(30 \%$ vs $25 \%, \mathrm{p}=0.39$, table 3$)$.

\section{Work lives of female doctors}

The median number of working hours of respondents was 42 hours per week (IQR 30-50), with 39 of those spent at work (table 3 ). The majority worked predominantly in public practices $(66 \%, 280 / 423)$, with fewer working in predominantly private practice $(24 \%, 102 / 423)$ or equal parts public and private practice $(10 \%, 41 / 423)$.
Women in cardiology compared with all other specialties were less likely to work predominantly in public practice ( $49 \%$ vs $69 \%, \mathrm{p}<0.001$ ). Women in cardiology worked longer hours than other specialties (median 42.5 hours vs 38 hours, $\mathrm{p}<0.001)$. Over half of all respondents were almost never on call $(49 \%, 206 / 423)$ and over one-third were only on call once per week $(36 \%, 152 / 423)$, though the amount of on call varied by specialty area. Women in cardiology were on call more often than women in other specialties, with $33 \%(19 / 57)$ on call more than once per week, compared with $12 \%(45 / 366)$ in other specialties $(\mathrm{p}<0.001)$.

Income was variable, with $16 \%(66 / 423)$ of respondents earning less than $\$ 100000$ per year, the majority (56\%, 233/423) between $\$ 100000$ and 200000 and $13 \%$ $(56 / 423)$ over $\$ 300000$. Women in cardiology were more likely to earn a higher income, with 35\% (19/57) earning over $\$ 300000$, compared with $10 \%(37 / 366)$ in all other specialties $(\mathrm{p}<0.001)$.

\section{Work-life balance and job satisfaction}

Almost three quarters $(307 / 412,75 \%)$ of participants reported that the demands of their work interfered with their home/family life, though fewer (171/412, $42 \%$ ) felt that their family responsibilities interfered with their work. The majority expressed that there was a lack of flexibility in their working hours, with only $28 \%(116 / 412)$ reporting that they were able to choose their working hours (table 4, figure 1). In regard to job satisfaction, almost all participants reported that they find their specialty professionally challenging $(400 / 413,97 \%)$ and intellectually stimulating $(405 / 412,98 \%)$ and $63 \%(260 / 413)$ felt that their specialty provided appropriate financial remuneration (table 4, figure 1 ).

There was variation in participants' perception of their specialty being 'female friendly' and 'family friendly', with women in cardiology significantly less 


\begin{tabular}{|c|c|c|c|c|c|c|c|c|c|c|}
\hline $\begin{array}{l}\text { Characteristic Number (\%) } \\
\text { unless stated otherwise }\end{array}$ & Cardiology & $\begin{array}{l}\text { Critical } \\
\text { care }\end{array}$ & GP & O\&G & Paediatrics & $\begin{array}{l}\text { Internal } \\
\text { medicine-other }\end{array}$ & Prevocational & Surgery & Other & Total \\
\hline Number of respondents & 57 & 68 & 80 & 15 & 36 & 117 & 29 & 11 & 39 & 452 \\
\hline \multicolumn{11}{|l|}{ Children } \\
\hline No & $17(30)$ & $19(28)$ & $8(10)$ & $3(20)$ & $8(22)$ & $35(30)$ & $23(79)$ & $15(38)$ & 2 (18) & $130(29)$ \\
\hline Yes & $40(70)$ & $49(72)$ & $72(90)$ & $12(80)$ & $28(78)$ & $82(70)$ & $6(21)$ & $24(62)$ & $9(82)$ & $322(71)$ \\
\hline \multicolumn{11}{|l|}{ Number of children } \\
\hline 0 & $17(30)$ & $19(28)$ & $8(10)$ & $3(20)$ & $8(22)$ & $35(30)$ & $23(79)$ & $15(38)$ & $2(18)$ & $130(29)$ \\
\hline $1-2$ & $29(51)$ & $38(56)$ & $54(68)$ & $9(60)$ & $23(64)$ & $73(62)$ & $6(21)$ & $17(44)$ & $6(55)$ & $255(56)$ \\
\hline 3 or more & $11(19)$ & $11(16)$ & $18(23)$ & $3(20)$ & $4(11)$ & $9(8)$ & $0(0)$ & $7(18)$ & $3(27)$ & $66(15)$ \\
\hline \multicolumn{11}{|l|}{ Stage of career for first child* } \\
\hline University & $1(3)$ & 2 (4) & $5(7)$ & $1(8)$ & $2(7)$ & $2(2)$ & $2(33)$ & $1(4)$ & $0(0)$ & $16(5)$ \\
\hline Internship & $1(3)$ & $0(0)$ & $4(6)$ & $1(8)$ & $0(0)$ & $1(1)$ & $2(33)$ & $0(0)$ & $0(0)$ & $9(3)$ \\
\hline Residency & $5(13)$ & $2(4)$ & $14(19)$ & $1(8)$ & $1(4)$ & $2(2)$ & $1(17)$ & $1(4)$ & $3(33)$ & $30(9)$ \\
\hline Specialty training & $5(13)$ & $27(55)$ & $18(25)$ & $6(50)$ & $18(64)$ & $46(56)$ & $\mathrm{N} / \mathrm{A}$ & $14(58)$ & $4(44)$ & $138(43)$ \\
\hline Fellowship & $9(23)$ & 2 (4) & $2(3)$ & $0(0)$ & $2(7)$ & $4(5)$ & $\mathrm{N} / \mathrm{A}$ & $2(8)$ & $1(11)$ & $22(7)$ \\
\hline $\mathrm{PhD}$ /research phase & $7(18)$ & $0(0)$ & $0(0)$ & $0(0)$ & $3(11)$ & $5(6)$ & $0(0)$ & $0(0)$ & $0(0)$ & $15(5)$ \\
\hline Consultant/fully qualified & $11(28)$ & $15(31)$ & $26(36)$ & $2(17)$ & $2(7)$ & $21(26)$ & $\mathrm{N} / \mathrm{A}$ & $7(29)$ & $1(11)$ & $85(26)$ \\
\hline \multicolumn{11}{|c|}{ Childcare $^{\star}$ (may select more than one option) } \\
\hline Nanny & $20(50)$ & $14(29)$ & $4(6)$ & $2(17)$ & $5(18)$ & $21(26)$ & $2(33)$ & $4(17)$ & $1(11)$ & $73(23)$ \\
\hline Day care & $13(33)$ & $25(51)$ & $40(56)$ & $9(75)$ & $18(64)$ & $47(57)$ & $2(33)$ & $13(54)$ & $5(56)$ & $172(53)$ \\
\hline Partner & $9(23)$ & $21(43)$ & $28(39)$ & $5(42)$ & $8(29)$ & 32 (39) & $4(67)$ & $8(33)$ & $4(44)$ & $119(37)$ \\
\hline Other family & $4(10)$ & $6(12)$ & $9(13)$ & $4(33)$ & $10(36)$ & $20(24)$ & $1(17)$ & $5(21)$ & $4(44)$ & $63(20)$ \\
\hline Other & $9(23)$ & $6(12)$ & $14(19)$ & $1(8)$ & $6(21)$ & $6(7)$ & $0(0)$ & $5(21)$ & $1(11)$ & $48(15)$ \\
\hline \multicolumn{11}{|l|}{ Return to work after child } \\
\hline Full-time clinical & $12(30)$ & $15(31)$ & $9(13)$ & $8(67)$ & $8(29)$ & $41(50)$ & $4(67)$ & $8(33)$ & $4(44)$ & 109 (34) \\
\hline Part-time clinical & $23(58)$ & $28(57)$ & $52(72)$ & $3(25)$ & $15(54)$ & $35(43)$ & $1(17)$ & $12(50)$ & $5(56)$ & $174(54)$ \\
\hline Non-clinical & $4(10)$ & $5(10)$ & $6(8)$ & $0(0)$ & $5(18)$ & $3(4)$ & $0(0)$ & $5(21)$ & $0(0)$ & $28(9)$ \\
\hline \multicolumn{11}{|c|}{ Interaction of career choices and family planning } \\
\hline Parenting delayed & $23(40)$ & $29(43)$ & $24(30)$ & $7(47)$ & $19(53)$ & $45(38)$ & $7(24)$ & $3(27)$ & $11(28)$ & $168(37)$ \\
\hline Training delayed & $9(16)$ & $20(29)$ & $14(18)$ & $4(27)$ & $16(44)$ & $33(28)$ & $4(14)$ & $4(36)$ & 7 (18) & $111(25)$ \\
\hline Chose no children & $1(2)$ & $1(1)$ & $0(0)$ & $0(0)$ & $1(3)$ & $1(1)$ & $0(0)$ & $0(0)$ & $0(0)$ & $4(1)$ \\
\hline Chose specialty & $6(11)$ & $18(26)$ & $58(73)$ & $1(7)$ & $16(44)$ & $42(36)$ & $8(28)$ & $1(9)$ & $16(41)$ & $166(37)$ \\
\hline Avoided changing positions & $1(2)$ & $15(22)$ & $7(9)$ & $4(27)$ & $9(25)$ & $18(15)$ & $4(14)$ & $0(0)$ & $5(13)$ & $63(14)$ \\
\hline
\end{tabular}

Due to the small numbers in each individual specialty group, statistical comparisons were performed for cardiology versus non-cardiology specialties in table 3.

*These sections are only relevant to those respondents who are parents, therefore proportions reflected as a percentage of those with children (in some instances, not all respondents with children answered each question).

GP, general practice; O\&G, obstetrics and gynaecology.

likely to agree that their specialty fits these descriptions (table 4, figure 1). Only 10/54 (19\%) of women in cardiology agreed that their specialty was 'female friendly' versus $267 / 358(75 \%)$ of women in other specialties, $\mathrm{p}<0.001$; similarly, $11 / 54(20 \%)$ of women in cardiology agreed that their specialty was 'family friendly', compared with 224/357 (63\%) in other specialties, $\mathrm{p}<0.001 . \mathrm{xx}$

\section{DISCUSSION}

The current study has several important findings. First, women in cardiology in Australia and NZ reported significantly poorer work-life balance, with greater hours worked and on-call commitments, compared with women in other specialties. In addition, women in cardiology were significantly less likely to perceive their specialty as being female friendly or family friendly. However, women in cardiology received significantly higher financial remuneration. These factors all play a role in career choice for female doctors and may represent reasons for the lack of gender diversity consistently reported within cardiology.

This study demonstrated that, compared with women in other specialties, women in cardiology worked longer hours, were more frequently on call and had poorer work-life balance. While the majority of female doctors felt that their work interfered with their home and family life, women in cardiology were significantly less likely to feel they led a balanced life. A key contributor to the interference of work with home and family life was in a lack of flexibility in working hours, reported by $63 \%$ 


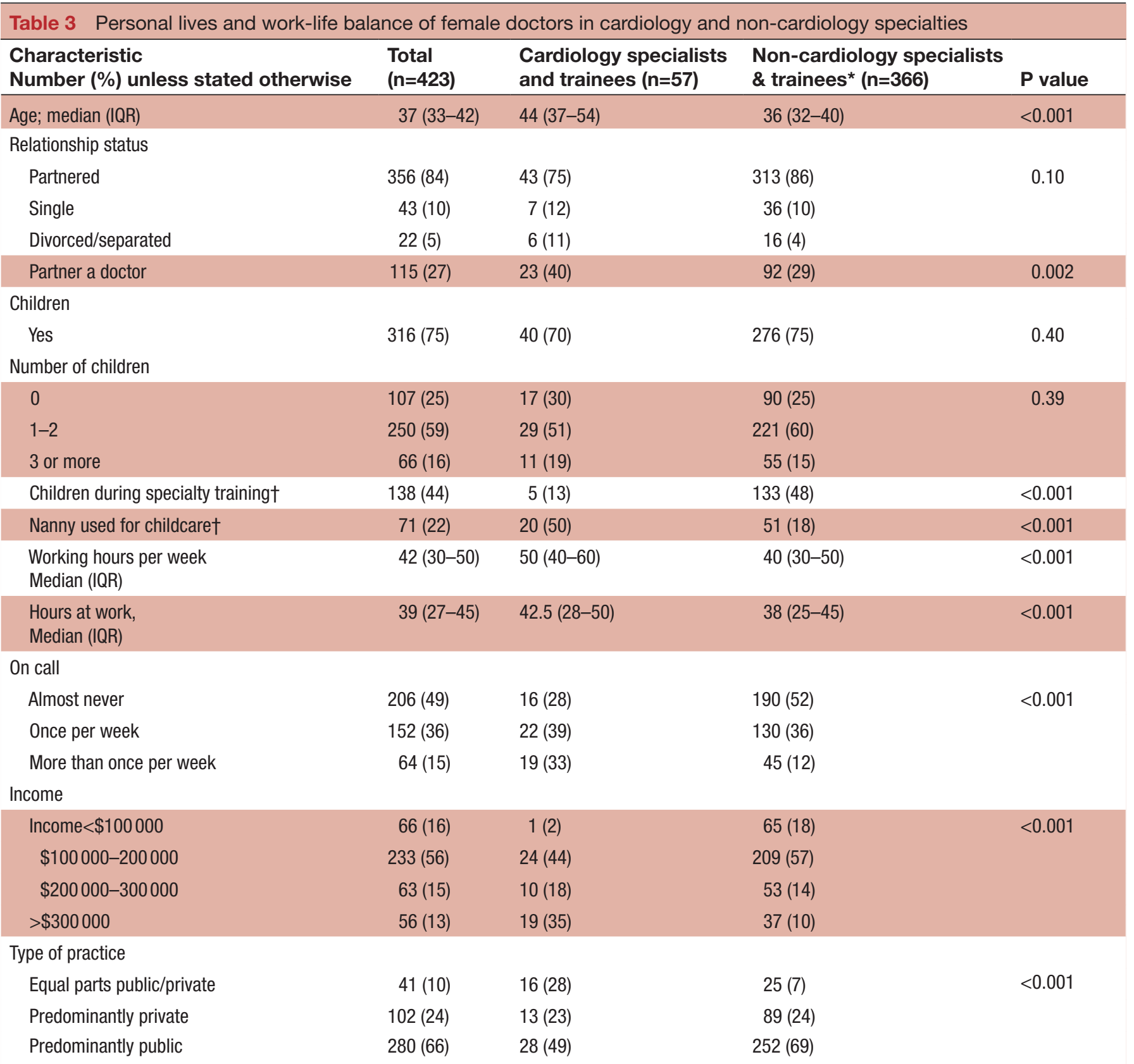

*Excludes prevocational doctors.

†These sections are only relevant to those respondents who are parents, therefore proportions reflected as a percentage of those with children (in some instances, not all respondents with children answered each question).

of respondents. Flexibility and predictability of working hours have both been previously identified as key issues for women in cardiology. ${ }^{17} 18$ An Australian Medical Association survey showed that the majority of doctors wanted greater access to flexible working hours, a demand that was similar across genders. ${ }^{19}$ Trainee doctors are increasingly placing greater emphasis on flexible training programmes which promote work-life balance when considering career choices. ${ }^{20}{ }^{21}$ Although historically women reported a greater influence of personal factors on decision-making, the difference between women and men appears to have diminished over time. ${ }^{22}$ Specialties with a favourable work-life balance and job flexibility are likely to be preferred, particularly for doctors with young families. $^{21}{ }^{23}$ Addressing work-life balance within cardiology is critical to attracting the best candidates to the specialty.

Although women in cardiology were as likely as women in other specialties to have children, they were more likely to report that their specialty was not family friendly or female friendly, similar to previous studies. ${ }^{24}$ In addition, $40 \%$ of women in cardiology stated they had delayed becoming a parent due to career choice and significantly lower proportions had their children during training. A previous study demonstrated that women in cardiology were more likely than men to experience discrimination 


\begin{tabular}{|c|c|c|c|c|}
\hline $\begin{array}{l}\text { Statement } \\
\text { Number (\%) unless stated otherwise }\end{array}$ & Total agree* $^{*}$ & $\begin{array}{l}\text { Cardiology } \\
\text { specialists and } \\
\text { trainees agree* }\end{array}$ & $\begin{array}{l}\text { Non-cardiology } \\
\text { specialists and } \\
\text { trainees agree* }\end{array}$ & $P$ value \\
\hline My specialty is female friendly & $277 / 412(67)$ & $10 / 54(19)$ & $267 / 358(75)$ & $<0.001$ \\
\hline My specialty is family friendly & $235 / 411(57)$ & $11 / 54(20)$ & $224 / 357(63)$ & $<0.001$ \\
\hline My specialty provides opportunity for career progression & $332 / 412(81)$ & $46 / 54(85)$ & $286 / 358(80)$ & 0.13 \\
\hline My specialty is intellectually stimulating & 405/412 (98) & $53 / 54(98)$ & $352 / 358(98)$ & 0.78 \\
\hline My specialty is professionally challenging & $400 / 413(97)$ & $53 / 55(96)$ & $347 / 358(97)$ & 0.61 \\
\hline My specialty provides appropriate financial remuneration & $260 / 413(63)$ & $48 / 55(87)$ & 212/358 (59) & $<0.001$ \\
\hline I am able to choose the start and finish time to my working day & $116 / 412(28)$ & $17 / 55(31)$ & $99 / 357(28)$ & 0.15 \\
\hline I feel I have enough time to do the lifestyle things I want to do & $103 / 413(25)$ & $13 / 55(24)$ & $90 / 358(25)$ & 0.63 \\
\hline My family responsibilities interfere with my work & $171 / 412(42)$ & $27 / 55(49)$ & $144 / 357(40)$ & 0.27 \\
\hline The demands of my work interfere with my home/family life & $307 / 412(75)$ & $43 / 55(78)$ & $265 / 358(74)$ & 0.56 \\
\hline I feel I have a pretty balanced life & 199/413 (48) & $18 / 55(33)$ & $181 / 358(51)$ & 0.03 \\
\hline My specialty is conducive to having/raising children & 236/412 (57) & $13 / 54(24)$ & $223 / 358(62)$ & $<0.001$ \\
\hline
\end{tabular}

*Proportion indicating 'agree' or 'strongly agree'.

on the basis of gender and parenting responsibilities. ${ }^{1725}$ Gender-based discrimination and sexual harassment has been reported within cardiology, but is often subtle and difficult to demonstrate. ${ }^{25}{ }^{26}$ Of note, a European study found that male respondents in the field of cardiology perceived women to be less capable of working in the field of interventional cardiology, describing 'work conditions considered too challenging, stressful, demanding and not suitable for women'. ${ }^{27}$ A recent US study showed that $47.8 \%$ of female cardiologists had experienced genderbased discrimination, mostly by colleagues, and $12 \%$ had experienced sexual harassment by a peer or colleague. ${ }^{26}$
A significant proportion of women in cardiology in the USA reported that family planning was raised during cardiology fellowship interviews. ${ }^{22}{ }^{28}$ Clear policies are needed, within each institution, to ensure gender-related and parenting-related discrimination does not take place.

Recent studies report that internal medicine trainees have an overwhelmingly negative perception of the culture within cardiology. ${ }^{21}{ }^{24}$ Moreover, these negative perceptions appeared to influence decisions regarding career choices including deciding against pursuing a career in cardiology. ${ }^{24}$ This study highlights the need to examine current training programmes and practices

$\leftarrow$ Other $\rightarrow$ Cardiology

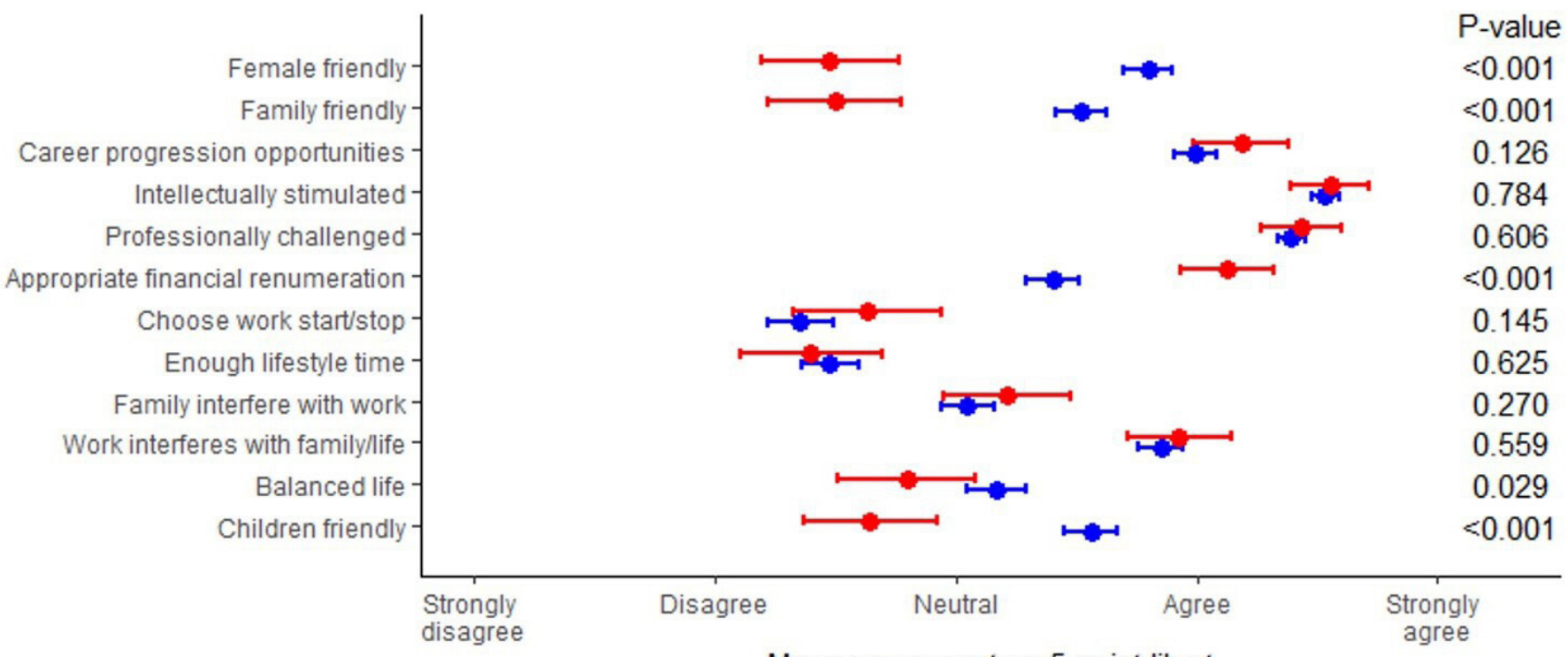

Mean agreement on 5-point likert

Figure 1 Cardiology and non-cardiology female doctors' perceptions of their own specialties; answers given on a 5-point Likert Scale (1=strongly disagree, 2=disagree, 3=neutral, 4=agree, 5=strongly agree). 
and promote innovations such as flexible training, stable hours and parental leave policies, to encourage more women into the field. ${ }^{17}$ Addressing identified issues of negative culture, constraints on personal lives and long hours may fortify a diverse cardiology workforce, reduce burn-out and enhance quality of patient care. ${ }^{24} 25$

We found that women in cardiology received significantly higher financial remuneration than women in other specialties, although this was not adjusted for number of hours worked, nor compared with the earnings of male doctors, previously shown to be higher than those of women. ${ }^{29}$ Interestingly, women in cardiology were more likely to incorporate private work into their practice. This raises the possibility that women in cardiology choose private practice to have a greater flexibility in working hours. The possibility that cardiology may not offer the same flexibility as other specialties in hospital settings should be explored. Medical students and junior doctors spend most of their training time within the hospital system, and the negative perceptions that they form of cardiology during this period likely contributes to not pursuing cardiology. ${ }^{21} 24$

This study showed that the majority of women in medicine were in a relationship and had children, with a similar proportion of women in cardiology partnered $(75 \%)$ and with children (70\%), compared with noncardiology doctors. This is in contrast to a US study that found women in cardiology were less likely than their male colleagues to be married and have children. ${ }^{17}$ While we found a numerically higher divorce/separation rate among women in cardiology compared with the noncardiology group, this did not reach statistical significance. Our survey showed that women in cardiology were more likely than women in other specialties to be partnered with another doctor.

The vast majority of women in our study, across all fields of medicine, felt they were professionally challenged and intellectually stimulated in their job. Encouragingly, the majority of women in cardiology, of whom $\sim 70 \%$ had children also agreed that they had career progression opportunities. This is in keeping with a US study that showed increasing levels of job satisfaction for women in cardiology over time. ${ }^{17}$ While the higher income and high levels of job satisfaction observed for women in cardiology in this study may partly counter some negative perceptions of a career in cardiology, much could also be gained by modifying training programmes and healthcare systems to create greater workplace flexibility. ${ }^{25}$ Innovations to promote and attract a more diverse workforce have the potential to improve outcomes for underrepresented patient groups and create a more inclusive culture for our trainees and colleagues. ${ }^{1011}$

\section{Limitations and future directions}

This study was limited by the relatively low response rate, likely the result of the survey being posted to Facebook groups where uptake is limited by number of views and active members. The anonymous nature of the survey resulted in an inability to limit survey attempts to one per person; however, responses were manually checked for duplication. Respondents were relatively young (median age 36 years) and predominantly Caucasian $(81 \%)$ or Asian (13\%). The young age likely reflects the method of recruitment via social media as well as the increasing proportion of women in medicine in more recent years. The ethnic profile of respondents is in keeping with Australian/NZ demographics; however, it limits generalisability to other geographic regions. A higher representation of women in cardiology was seen due to specifically targeting this cohort via email and as a result, women in cardiology were older, potentially creating a bias towards higher income and proportion in private practice. Due to the relatively small numbers of respondents from each individual specialty, we could only compare cardiology to non-cardiology specialties combined in order to maintain statistical power. Similarly, the number of participants was not adequate for examination of subgroups by stage of training. Our study was targeted to female doctors, and hence we cannot comment on differences in work-life balance between genders. Our survey assessed only quantitative results. Future research could include qualitative and/or interview components to obtain other perspectives and expand on the results obtained by this study.

\section{CONCLUSIONS}

Women in medicine in Australia and NZ report high job satisfaction but encounter significant barriers to work-life balance, with the majority reporting the demands of work interfere with home and family life. Women in cardiology experience significantly poorer work-life balance compared with other specialties, including longer working hours, more on call and a less family-friendly and female-friendly culture. This provides important insights into barriers encountered by women in medicine, particularly in cardiology, and may help inform efforts to improve recruitment of female trainees.

\section{Author affiliations}

${ }^{1}$ Department of Cardiology, Northern Health, Epping, Victoria, Australia

${ }^{2}$ Melbourne Medical School, University of Melbourne, Melbourne, Victoria, Australia

${ }^{3}$ Department of Cardiology, Nepean Hospital, Penrith, New South Wales, Australia

${ }^{4}$ Faculty of Medicine, University of New South Wales, Sydney, New South Wales,

Australia

${ }^{5}$ Sydney School of Medicine, University of Sydney, Sydney, New South Wales,

Australia

${ }^{6}$ Biostatistics, NHMRC Clinical Trials Centre, Camperdown, New South Wales, Australia

${ }^{7}$ Department of Cardiology, The Alfred Hospital, Melbourne, Victoria, Australia ${ }^{8}$ Baker Heart \& Diabetes Institute, Melbourne, Victoria, Australia

${ }^{9}$ Department of Medicine, Nursing and Health Sciences, Monash University, Clayton, Victoria, Australia

${ }^{10}$ Department of Cardiology, Barwon Health, Geelong, Victoria, Australia

${ }^{11}$ Department of Cardiology, Cabrini Health, Malvern, Victoria, Australia

${ }^{12}$ Westmead Applied Research Centre, University of Sydney, Sydney, New South

Wales, Australia

${ }^{13}$ Department of Cardiology, Westmead Hospital, Westmead, New South Wales, Australia

Twitter Anastasia Vlachadis Castles @a_v_castles 
Contributors All authors were involved in the design of the study, survey and results reporting and have approved the final submission. KR and AVC performed statistical analyses. AVC and SZ are guarantors responsible for the overall content.

Funding The authors have not declared a specific grant for this research from any funding agency in the public, commercial or not-for-profit sectors.

Competing interests None declared.

Patient consent for publication Not required.

Ethics approval The study was approved by the Monash Health Human Research Ethics Committee with no consent required. All research activity was performed in accordance with the Declaration of Helsinki and participant involvement was voluntary with no identifiable information collected.

Provenance and peer review Not commissioned; externally peer reviewed.

Data availability statement Data are available upon reasonable request. Deidentified participant data are available by contacting AVC (ORCID 0000-00025123-5980). The data can be used at the discretion of the authors.

Open access This is an open access article distributed in accordance with the Creative Commons Attribution Non Commercial (CC BY-NC 4.0) license, which permits others to distribute, remix, adapt, build upon this work non-commercially, and license their derivative works on different terms, provided the original work is properly cited, appropriate credit is given, any changes made indicated, and the use is non-commercial. See: http://creativecommons.org/licenses/by-nc/4.0/.

\section{ORCID iDs}

Anastasia Vlachadis Castles http://orcid.org/0000-0002-5123-5980

Anna L Beale http://orcid.org/0000-0002-3802-1961

Louise Segan http://orcid.org/0000-0001-5585-6146

Sarah Gutman http://orcid.org/0000-0002-2294-6142

Sarah Zaman http://orcid.org/0000-0001-6289-583X

\section{REFERENCES}

1 Australian Government, Department of Health. Medical training review panel: 19th report; 2016.

2 Burgess S, Shaw E, Ellenberger K, et al. Women in medicine: addressing the gender gap in interventional cardiology. J Am Coll Cardiol 2018;72): :2663-7.

3 Moberly T. Men outnumber women three to one in some specialties. BMJ 2018;363:k4098.

4 Moe TG. Pregnancy in fellowship: building a career and family. J Am Coll Cardiol 2014:64:734-6.

5 Segan L, Vlachadis Castles A. Women in cardiology in Australia-Are we making any progress? Heart Lung Circ 2019;28:690-6.

6 Wenger NK. Women in cardiology: the US experience. Heart 2005;91:277-9.

7 Andreotti F, Crea F. Women in cardiology: a European perspective. Heart 2005;91:275-6.

8 Timmis AD, English KM. Women in cardiology: a UK perspective. Heart 2005;91:273-4.

9 Medical deans Australia and New Zealand. 2016 medical students statistics; 2016.
10 Curtis AB, Rodriguez F. Choosing a career in cardiology: where are the women? JAMA Cardiol 2018;3:691-2.

11 Nivet MA. Commentary: diversity 3.0: a necessary systems upgrade. Acad Med 2011:86:1487-9.

12 Baumhäkel M, Müller U, Böhm M. Influence of gender of physicians and patients on guideline-recommended treatment of chronic heart failure in a cross-sectional study. Eur J Heart Fail 2009;11:299-303.

13 Greenwood BN, Carnahan S, Huang L. Patient-Physician gender concordance and increased mortality among female heart attack patients. Proc Natl Acad Sci U S A 2018;115:8569-74.

14 Kalliath T, Brough P. Work-life balance: a review of the meaning of the balance construct. Journal of Management \& Organization 2008;14:323-7.

15 Starmer AJ, Frintner MP, Matos K, et al. Gender discrepancies related to pediatrician work-life balance and household responsibilities. Pediatrics 2019;144. doi:10.1542/peds.2018-2926. [Epub ahead of print: 1009 2019].

16 R Core Team. R: a language and environment for statistical computing, 2020. Available: https://www.R-project.org/

17 Poppas A, Cummings J, Dorbala S, et al. Survey results: a decade of change in professional life in cardiology: a 2008 report of the ACC women in cardiology Council. J Am Coll Cardiol 2008;52:2215-26.

18 Hofäcker D, König S. Flexibility and work-life conflict in times of crisis: a gender perspective. Int J Sociol Soc Policy 2013;33:613-35.

19 Australian Medical Association. Ama work-life flexibility survey. Australian Capital Terriitory AMA National; 2008.

20 Jovic E, Wallace JE, Lemaire J. The generation and gender shifts in medicine: an exploratory survey of internal medicine physicians. BMC Health Serv Res 2006;6:55.

21 Kurdi $\mathrm{H}$, Morgan $\mathrm{H}$, Williams $\mathrm{C}$. Women not in cardiology: where are we going wrong? A survey of the perceptions and barriers to training. Br J Cardiol 2020;27.

22 Lewis SJ, Mehta LS, Douglas PS, et al. Changes in the professional lives of cardiologists over 2 decades. J Am Coll Cardiol 2017:69:452-62.

23 Yang Y, Li J, Wu X, et al. Factors influencing subspecialty choice among medical students: a systematic review and meta-analysis. BMJ Open 2019;9:e022097.

24 Douglas PS, Rzeszut AK, Bairey Merz CN, et al. Career preferences and perceptions of cardiology among US internal medicine trainees: factors influencing cardiology career choice. JAMA Cardiol 2018;3:682-691.

25 Lau ES, Wood MJ. How do we attract and retain women in cardiology? Clin Cardiol 2018;41:264-8.

26 Mehta L, Sharma G, Douglas P, et al. Discrimination and harrassment in cardiology: insights from the 2019 American College of cardiology global professional life survey. J Am Coll Cardiol 2020;75:3631.

27 Capranzano P, Kunadian V, Mauri J, et al. Motivations for and barriers to choosing an interventional cardiology career path: results from the EAPCI women Committee worldwide survey. Eurolntervention 2016;12:53-9.

28 Mwakyanjala EJ, Cowart JB, Hayes SN, et al. Pregnancy and parenting during cardiology fellowship. J Am Heart Assoc 2019;8:e012137.

29 Jagsi R, Biga C, Poppas A, et al. Work activities and compensation of male and female cardiologists. J Am Coll Cardiol 2016;67:529-41. 